\title{
Spectrum of Clinical Research in Juvenile Idiopathic Arthritis: A Cross-Sectional Analysis of Registered Studies in Clinicaltrials.gov and Clinicaltrialsregister.eu
}

\author{
Ronny Lehmann ${ }^{1}$ (D) and Markus Ries ${ }^{2,3,4, *(\mathbb{D})}$ \\ 1 Center for Pediatric and Adolescent Medicine, Department of General Pediatrics I, Im Neuenheimer Feld 430, \\ 69120 Heidelberg, Germany; ronny.lehmann@med.uni-heidelberg.de \\ 2 Center for Pediatric and Adolescent Medicine, Department of Pediatric Neurology and Metabolic Medicine, \\ Im Neuenheimer Feld 430, 69120 Heidelberg, Germany \\ 3 Center for Rare Disorders, Heidelberg University Hospital, Im Neuenheimer Feld 430, \\ 69120 Heidelberg, Germany \\ 4 Center for Virtual Patients, Medical Faculty, University of Heidelberg, Im Neuenheimer Feld 430, \\ 69120 Heidelberg, Germany \\ * Correspondence: markus.ries@uni-heidelberg.de
}

check for updates

Citation: Lehmann, R.; Ries, M. Spectrum of Clinical Research in Juvenile Idiopathic Arthritis: A

Cross-Sectional Analysis of

Registered Studies in

Clinicaltrials.gov and

Clinicaltrialsregister.eu. Biomedicines

2021, 9, 1860. https://doi.org/

$10.3390 /$ biomedicines 9121860

Academic Editor: Michal Tomcik

Received: 25 October 2021

Accepted: 5 December 2021

Published: 8 December 2021

Publisher's Note: MDPI stays neutral with regard to jurisdictional claims in published maps and institutional affiliations.

Copyright: (c) 2021 by the authors. Licensee MDPI, Basel, Switzerland. This article is an open access article distributed under the terms and conditions of the Creative Commons Attribution (CC BY) license (https:// creativecommons.org/licenses/by/ $4.0 /)$

\begin{abstract}
The management of juvenile idiopathic arthritis (JIA) has improved tremendously in recent years due to the introduction of new drug therapies but remains complex in terms of nonpharmaceutical issues. In order to determine the direction of scientific progress by characterizing the current spectrum of ongoing clinical research in JIA, we analyzed all ongoing studies in the field of JIA-registered in clinicaltrials.gov and clinicaltrialsregister.eu-concerning sponsoring, enrollment, duration, localization, and particularly objectives. The close of the database was 7 January 2021. After identifying double-registered studies, $n=72$ went into further analysis. Of these, $61.1 \%$ were academia-sponsored and $37.5 \%$ were sponsored by the pharma industry. The majority of the studies was of the interventional type (77.8\%), while others $(22.2 \%)$ were observational. The median planned enrollments were 100 participants (interventional studies) and 175 participants (observational studies), respectively. The duration differed remarkably from one month to more than 15 years, with a median of 42.5 months. A total of $61.1 \%$ of studies were located in a single country, and $38.9 \%$ were in several. Europe and North America clearly dominated the study localizations. The study objectives were DMARDs (56.9\%), followed by diagnostics and disease activity measurement $(18.1 \%)$, and medication other than DMARD (12.5\%), besides others. Studies on DMARDs were mainly sponsored by industry, predominantly interventional studies on established and novel biologics, with several on specific issues such as systemic JIA and others. The spectrum of registered studies is currently centered on drug therapy and diagnostics, while other issues in JIA play a subordinated role in current research. Drug development was transferred from adult rheumatology into the JIA population with little innovation for children. Future research should take specific pediatric needs better into account.
\end{abstract}

Keywords: juvenile idiopathic arthritis; research registry; clinical trial; DMARD

\section{Introduction}

Juvenile idiopathic arthritis (JIA) is one of the most prevalent chronic diseases in childhood, with 16-150 cases per 100,000 population in developed countries [1]. Undertreated JIA results in joint corrosion, reduced quality of life and participation, and may cause persistent disabilities [2-4]. Furthermore, pediatric patients are at risk of local growth disturbances, (general) growth failure, and pubertal disorders [1,5]. JIA-associated uveitis and temporomandibular joint involvement are prevalent challenging treatment issues [2,6].

The management of JIA has tremendously improved over the last decades [7]. Molecular-immunology studies have led to a better understanding of etiologies and patho- 
geneses [3]. The introduction of biological and targeted synthetic disease modifying antirheumatic drugs (DMARDs) revolutionized the treatment and outcomes of JIA patients and will likely be applied in personalized treatment strategies [2,4]. Scientific research and drug development are brought into practice through the structural establishment of pediatric rheumatology networks and disease registers, and emerging guidelines for JIA [1,2,8-12].

Despite these considerable advancements, the treatment of JIA remains complex and improvable. Better definitions of disease entities and their pathogeneses are needed for improved classification and treatment strategies $[1,8]$, as well as specific biomarkers for personalized treatment tuning $[2,8,13,14]$. Pediatric-approved DMARDs require long-term observation through registry studies [15], and recently approved DMARDs from adult medicine-i.e., in the treatment of rheumatoid arthritis-need to be explored for their potentials and risks in pediatric patients with JIA [16]. Novel drugs that selectively target the molecules or pathways involved in inflammation are needed to offer new treatment perspectives in refractory cases; therefore, prospective clinical studies are inevitable [2,8].

However, improving pediatric rheumatologic care is more than improving pediatric pharmacological care. A nontrivial question is: how can individual access to pediatric rheumatologic care be provided for children with such diseases [17,18]? Pediatric-specific issues (family-centered care, social integration and rehabilitation, etc.) must be addressed, as well as transition, as key issues of every chronic disease in childhood [19].

Against the background of JIA-associated and pediatric-specific health issues, as well as recent improvements in therapy and diagnostics, and known research needs, the purpose of this study is to characterize current clinical research in the field of JIA.

\section{Materials and Methods}

\subsection{Search for Ongoing Clinical Studies}

Web-based databases of the U.S. National Library of Medicine (clinicaltrials.gov) and the European Union Clinical Trials Register (clinicaltrialsregister.eu) were assessed on 7 January 2021 for ongoing clinical studies, with the search keywords "juvenile idiopathic arthritis" and the synonyms "JIA" and "juvenile chronic arthritis". Filters were applied for age range (all age groups under 18 years) and study status. The term 'ongoing' refers to not yet finally completed studies at the time of analysis, including studies with the status "Recruiting", "Not yet recruiting", "Active/not recruiting", "Enrolling by invitation", "Suspended", "Ongoing", "Restarted", and "Temporarily halted", respectively. Data were downloaded for further analysis.

STROBE criteria (Strengthening the Reporting of Observational studies in Epidemiology) were applied for the design, conduction, and reporting of this study [20].

\subsection{Data Analysis}

Microsoft Excel 2019 MSO, Edmond WA, USA, was used for data analysis. Standard techniques for descriptive statistics were applied. Study titles and description details were analyzed concerning sponsor, enrollment, duration, localization of study centers, and study type and objectives. Double-registered studies were identified and doublets were excluded. Missing data were not imputed. The sponsor was categorized into either industry or academia (including universities, public institutions and hospitals). Planned enrollment of participants was also extracted from description details. By the start date, ongoing "duration" of studies was calculated in months using the earlier date in the case of doublets in both registries. For localization of study centers, we displayed the top five locations for single- and multi-country studies, respectively, for which countries were clustered to their super-ordinated medical authorities (i.e., EU countries-EMA). Study details were analyzed for classification of interventional or observational studies, and their clinical phases, where appropriate. For the determination of study objectives, keywords were generated from study descriptions, and content analysis was used to determine answer categories [21]. 


\section{Results}

\subsection{Registered Studies}

Overall, $n=56$ studies registered on clinicaltrials.gov and $n=34$ studies on clincialtrialsregister.eu met the search criteria. Of these, $n=18$ studies were identified as being double-registered. The contents of $n=72$ studies were further analyzed. In the following passage, we present the main results. For more details, see Supplementary File S1.

\subsection{General Findings}

\subsubsection{Sponsor}

Academia sponsored $44 / 72(61.1 \%)$ of the found studies, and industry sponsored $27 / 72(37.5 \%)$. One study was mixed sponsored. Of the academia-sponsored studies, $3 / 44$ $(6.8 \%)$ were doubled-registered in both registers, as were $15 / 27(55.6 \%)$ of the industrysponsored studies.

\subsubsection{Planned Enrollment}

For the interventional studies, the planned enrollment was median 100, with a minimum of 6 and a maximum of 340 participants. Planned enrollment for the observational studies was median 175, with a minimum of 10 and a maximum of 9000 participants.

\subsubsection{Duration of Studies}

The start dates of $n=2$ studies were given in the future at the time of assessment and were therefore not used for the calculation of duration. The duration of ongoing studies was calculated from $n=70$ studies with a median of 42.5 months, a minimum of 1 month and a maximum of 183 months (more than 15 years). This means half of the ongoing studies started in late 2017 or thereafter. The start dates of the other half varied between 2005 and the first half of 2017.

\subsubsection{Locations}

Of the analyzed studies, $44 / 72(61.1 \%)$ were located in a single country, and 28/72 $(38.9 \%)$ in multiple countries. The most frequent countries for a single location were: France $(9 / 44)$, the Netherlands (6/44), the United States of America (6/44), Canada (5/44), China $(3 / 44)$, and Italy $(3 / 44)$. When multiple countries were involved, the most frequent countries were: EU countries (24/28), the United Kingdom (17/28), Russian Federation (14/28), Mexico (13/28), and the United States of America (13/28). Geographically, European countries were involved in 54/72 studies (75.0\%), North American countries in 30/72 studies (41.7\%), South American and Asian countries in 14/72 studies (19.4\%) each, African countries in 8/72 studies (11.1\%), and Australia and Oceanian countries in 6/72 studies $(8.3 \%)$.

\subsubsection{Study Types}

The found study type was interventional in 56/72 (77.8\%) and observational in 16/72 (22.2\%) studies. A clinical phase was given in $N=44 / 56$ interventional studies:

- Phase I: 5 studies,

- Phase II: 4 studies,

- $\quad$ Phases I+II: 3 studies,

- Phase III: 22 studies,

- $\quad$ Phase IV: 10 studies.

\subsection{Study Objectives}

For the proportions of study objectives, see also Figure 1. 


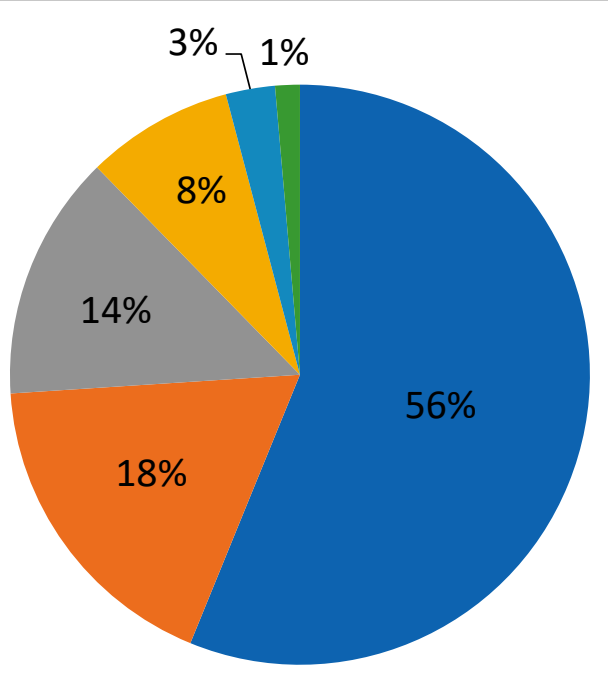

DMARD agents

Diagnostics and disease activity

non-DMARD medication

non-medication

treatment

vaccination

COVID-19 pandemic

Figure 1. Proportions of study objectives.

\subsubsection{DMARDs}

A total of $41 / 72(56.9 \%)$ studies were related to DMARDs in the fields of JIA, including JIA-associated uveitis. Industry sponsors were involved in 27/41 studies. A total of 14/41 studies were sponsored by academia only. Studies addressed conventional synthetic DMARDs (csDMARDs; 10/41 studies), biological DMARDs (bDMARDs; $29 / 41$ studies, hereof six studies also including csDMARDs), and targeted synthetic DMARDs (tsDMARDs; 8/41 studies). Conversely, 31/41 studies on DMARDs did not involve any csDMARD as a variable or control, and only $4 / 41$ studies did not involve any bDMARD or tsDMARD, as seen in Figure 2. Biosimilar DMARDs were not involved.

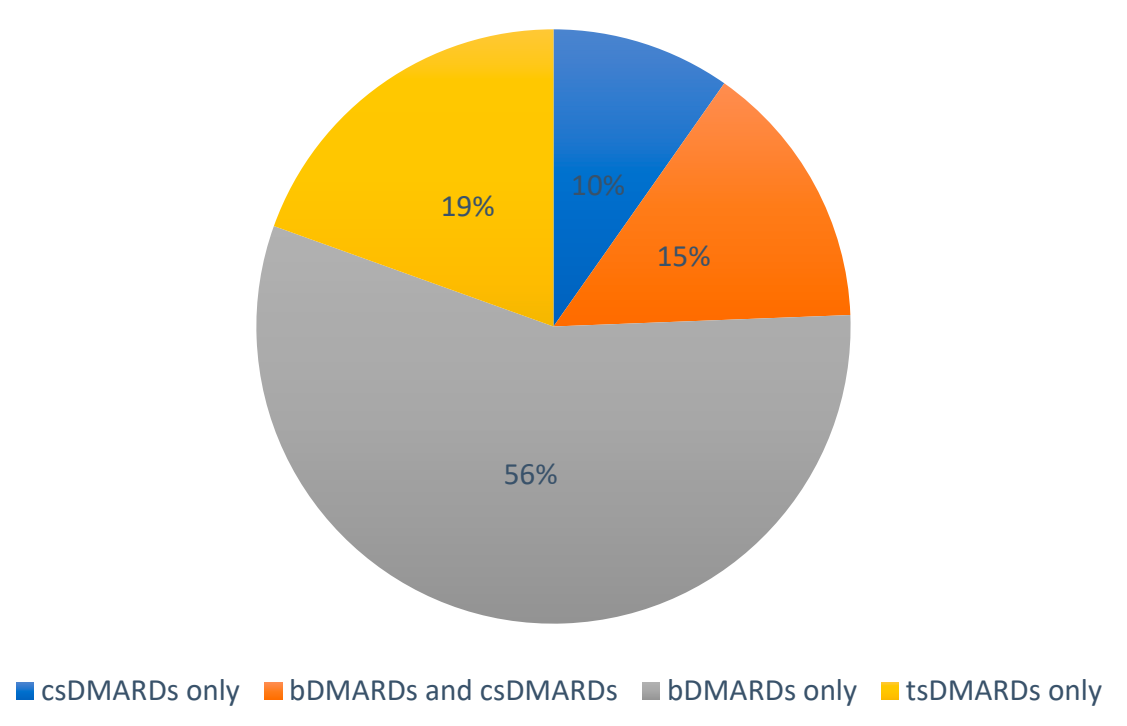

Figure 2. Proportions of DMARD agents involved in registered studies.

Only 5/41 studies were of the observational type, while all others were interventional. The following DMARDs were specifically studied in these studies, in descending order (partly multiple agents involved per study): 
- Methotrexate (MTX; 10/41 studies, hereof three observational studies),

- $\quad$ Abatacept (ABA; 6/41 studies, hereof one observational study),

- $\quad$ Etanercept (ETA; 6/41 studies),

- Tocilizumab (TOC; 6/41 studies),

- Adalimumab (ADA; $4 / 41$ studies, hereof one observational study),

- $\quad$ Baricitinib (BAR; $4 / 41$ studies),

- $\quad$ Tofacitinib (TOF; 3/41 studies),

- Canakinumab (CAN; $2 / 41$ studies),

- Golimumab (GOL; 2/41 studies, hereof one observational study),

- Sarilumab (SAR; 2/41 studies),

- $\quad$ Secukinumab (SEC; $2 / 41$ studies),

- Anakinra (ANA; 1/41 study),

- Certolizumab (CER; 1/41 study),

- Hydroxychloroquine (HCQ; 1/41 study),

- $\quad$ Ixekizumab (IXE; $1 / 41$ study),

- $\quad$ Sulfasalazine (SUL; $1 / 41$ study),

- $\quad$ Upadacitinib (UPA; $1 / 41$ study).

Of the studied biological and targeted synthetic agents, the corresponding targets are shown in Figure 3.

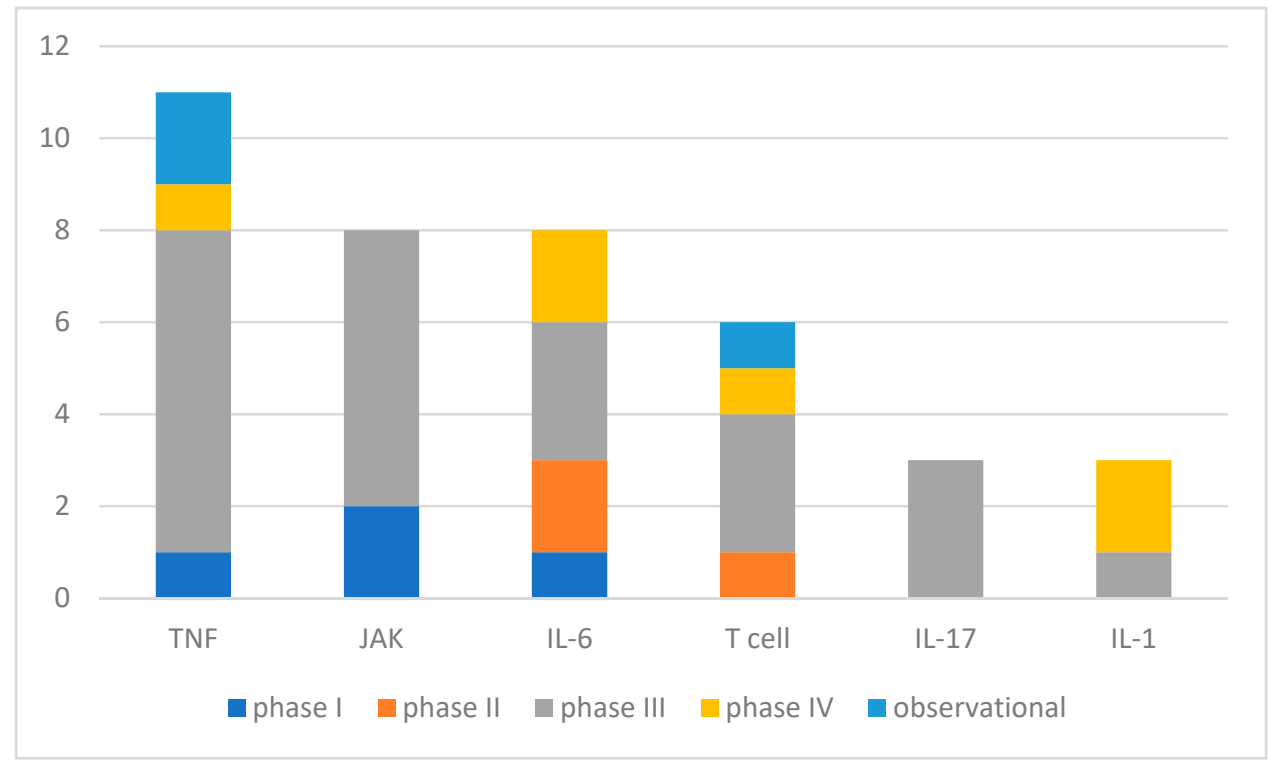

Figure 3. Number of studies concerning bDMARDs and tsDMARDs sorted for biological targets (interventional and observational studies, partly multiple agents involved per study). IL-interleukine, JAK-janus kinase, TNF-tumor necrosis factor.

The enrollment of observational studies was median 833, with a minimum of 10 and a maximum of 9000 . For clinical phases and planned enrollments in the interventional studies on DMARDs, see Tables 1 and 2. All industry-sponsored studies were located in multiple countries, whereas only two of the academia-sponsored studies had locations in more than one country (the USA, UK, and several EU countries, respectively). 
Table 1. Registered interventional studies involving csDMARD agents, clinical phases, and planned enrollments. * refers to a study involving multiple csDMARDs. sJIA—systemic JIA.

\begin{tabular}{|c|c|c|c|c|c|c|c|}
\hline Agent & Phase & Registration Number & Enroll. & $\begin{array}{c}\text { Age } \\
\text { (Years) }\end{array}$ & $\begin{array}{l}\text { Specifically } \\
\text { Addresses }\end{array}$ & $\begin{array}{l}\text { Start } \\
\text { Year }\end{array}$ & $\begin{array}{l}\text { bDMARDs } \\
\text { Involved }\end{array}$ \\
\hline \multirow{7}{*}{ MTX } & II & EudraCT2008-006741-70 & 252 & $0-17$ & & 2008 & $\mathrm{n} / \mathrm{a}$ \\
\hline & III & NCT02277444 & 130 & $2-17$ & & 2014 & GOL \\
\hline & III & NCT03728478 & 260 & $2-17$ & withdrawal & 2019 & ETA \\
\hline & III & EudraCT2005-001086-34 & 200 & $2-17$ & withdrawal & 2005 & $\mathrm{n} / \mathrm{a}$ \\
\hline & IV & NCT03301883 & 74 & $2-17$ & sJIA & 2018 & TOC \\
\hline & IV & EudraCT2013-003956-18 & 325 & $1-17$ & withdrawal & 2014 & $\begin{array}{l}\text { ABA, ADA, } \\
\text { ETA }\end{array}$ \\
\hline & IV & EudraCT2014-003260-20* & 130 & $2-17$ & & 2016 & $\mathrm{n} / \mathrm{a}$ \\
\hline HCQ & IV & EudraCT2014-003260-20 * & 130 & $2-17$ & & 2016 & $\mathrm{n} / \mathrm{a}$ \\
\hline SUL & IV & EudraCT2014-003260-20 * & 130 & $2-17$ & & 2016 & $\mathrm{n} / \mathrm{a}$ \\
\hline
\end{tabular}

Table 2. Registered interventional studies involving bDMARD and tsDMARD agents, clinical phases, and planned enrollments. *,+ refer to studies involving multiple bDMARDs. Start year in brackets refers to studies with start date in future of data acquisition. ERA—enthesitis-related arthritis, ILinterleukine, JAK—janus kinase, JPsA—juvenile psoriatic arthritis, sJIA—systemic JIA, TNF—tumor necrosis factor.

\begin{tabular}{|c|c|c|c|c|c|c|c|}
\hline Target & Agent & Phase & Registration Number & Enroll. & $\begin{array}{c}\text { Age } \\
\text { (Years) }\end{array}$ & $\begin{array}{l}\text { Specifically } \\
\text { Addresses }\end{array}$ & $\begin{array}{l}\text { Start } \\
\text { Year }\end{array}$ \\
\hline \multirow{8}{*}{ TNF } & \multirow{3}{*}{$\mathrm{ADA}$} & \multirow{2}{*}{ III } & NCT02840175* & 62 & $2-17$ & withdrawal & 2017 \\
\hline & & & NCT03816397 & 118 & $2-17$ & uveitis & 2020 \\
\hline & & IV & EudraCT2013-003956-18 † & 325 & $1-17$ & withdrawal & 2014 \\
\hline & \multirow{3}{*}{ ETA } & I & NCT04585711 & 30 & $2-65$ & & (2022) \\
\hline & & III & $\begin{array}{c}\text { NCT01421069 } \\
\text { NCT02840175* } \\
\text { NCT03728478 } \\
\text { EudraCT2009-012520-84 }\end{array}$ & $\begin{array}{c}109 \\
62 \\
260 \\
100\end{array}$ & $\begin{array}{l}2-30 \\
2-17 \\
2-17 \\
2-17\end{array}$ & $\begin{array}{l}\text { ERA, JPsA } \\
\text { withdrawal } \\
\text { withdrawal } \\
\text { ERA, JPsA }\end{array}$ & $\begin{array}{l}2011 \\
2017 \\
2019 \\
2009\end{array}$ \\
\hline & & IV & EudraCT2013-003956-18† & 325 & $1-17$ & withdrawal & 2014 \\
\hline & CER & III & NCT01550003 & 193 & $2-17$ & & 2012 \\
\hline & GOL & III & NCT02277444 & 130 & $2-17$ & & 2014 \\
\hline \multirow{4}{*}{ JAK } & BAR & III & $\begin{array}{l}\text { NCT03773965 } \\
\text { NCT03773978 } \\
\text { NCT04088396 } \\
\text { NCT04088409 }\end{array}$ & $\begin{array}{c}190 \\
197 \\
103 \\
40\end{array}$ & $\begin{array}{l}1-17 \\
2-17 \\
1-17 \\
2-17\end{array}$ & $\begin{array}{l}\text { sJIA } \\
\text { uveitis }\end{array}$ & $\begin{array}{l}2019 \\
2018 \\
2020 \\
2019\end{array}$ \\
\hline & \multirow[b]{2}{*}{ TOF } & I & EudraCT2011-004914-40 & 24 & $2-17$ & & 2012 \\
\hline & & III & $\begin{array}{l}\text { NCT01500551 } \\
\text { NCT03000439 }\end{array}$ & $\begin{array}{l}340 \\
100\end{array}$ & $\begin{array}{l}2-18 \\
2-17\end{array}$ & sJIA & $\begin{array}{l}2013 \\
2018\end{array}$ \\
\hline & UPA & I & NCT03725007 & 54 & $2-17$ & & 2019 \\
\hline \multirow{5}{*}{ IL-6 } & SAR & II & $\begin{array}{l}\text { NCT02776735 } \\
\text { NCT02991469 }\end{array}$ & $\begin{array}{c}100 \\
72\end{array}$ & $\begin{array}{l}2-17 \\
1-17\end{array}$ & sJIA & $\begin{array}{l}2016 \\
2018\end{array}$ \\
\hline & \multirow{4}{*}{ TOC } & I & NCT02165345 & 82 & $2-18$ & sJIA & 2014 \\
\hline & & III & $\begin{array}{l}\text { NCT02840175* } \\
\text { EudraCT2007-000872-18 } \\
\text { EudraCT2009-011593-15 }\end{array}$ & $\begin{array}{c}62 \\
108 \\
185\end{array}$ & $\begin{array}{l}2-17 \\
2-17 \\
2-17 \\
\end{array}$ & $\begin{array}{l}\text { withdrawal } \\
\text { SJIA } \\
\text { withdrawal }\end{array}$ & $\begin{array}{l}2017 \\
2008 \\
2009\end{array}$ \\
\hline & & \multirow{2}{*}{ IV } & NCT03301883 & 74 & $2-17$ & sJIA & 2018 \\
\hline & & & EudraCT2012-000444-10 & 43 & $2-17$ & sJIA & 2012 \\
\hline \multirow{3}{*}{$\mathrm{T}$ cell } & \multirow{3}{*}{$\mathrm{ABA}$} & I/II & NCT03733067 & 40 & $8-65$ & & (2021) \\
\hline & & III & $\begin{array}{l}\text { NCT01844518 } \\
\text { NCT02840175* } \\
\text { NCT03841357 }\end{array}$ & $\begin{array}{c}187 \\
62 \\
306\end{array}$ & $\begin{array}{l}2-17 \\
2-17 \\
2-16\end{array}$ & withdrawal & $\begin{array}{l}2013 \\
2017 \\
2019\end{array}$ \\
\hline & & IV & EudraCT2013-003956-18 † & 325 & $1-17$ & withdrawal & 2014 \\
\hline
\end{tabular}


Table 2. Cont.

\begin{tabular}{ccccccccc}
\hline \multirow{2}{*}{ Target } & Agent & Phase & Registration Number & Enroll. & $\begin{array}{c}\text { Age } \\
\text { (Years) }\end{array}$ & $\begin{array}{c}\text { Specifically } \\
\text { Addresses }\end{array}$ & $\begin{array}{c}\text { Start } \\
\text { Year }\end{array}$ \\
\hline \multirow{3}{*}{ IL-17 } & IXE & III & NCT04527380 & 100 & $2-17$ & ERA, JPsA & 2021 \\
\cline { 2 - 9 } & \multirow{2}{*}{ SEC } & III & EudraCT2016-003761-26 & 80 & $2-99$ & ERA, JPsA & 2019 \\
& ANA & IV & EudraCT2015-004393-16 & 55 & $2-17$ & sJIA & 2016 \\
\hline \multirow{2}{*}{ IL-1 } & \multirow{2}{*}{ CAN } & III & EudraCT2008-005476-27 & 122 & $2-99$ & sJIA & 2009 \\
\cline { 2 - 9 } & & IV & EudraCT2018-004284-30 & 20 & $2-17$ & sJIA & 2020 \\
\hline
\end{tabular}

\subsubsection{Diagnostics and Measurement of Disease Activity}

A total of $13 / 72(18.1 \%)$ studies were related to diagnostics and disease activity in JIA in a broader sense. All of these studies were academia-sponsored and located in a single country. Studies concerned musculoskeletal and bone health, differential diagnosis of septic arthritis, imaging of arthritis, and temporomandibular involvement, as well as etiology and pathogenesis of systemic JIA, disease activity biomarker, and a national disease registry. The median enrollment of these studies was 90, with a minimum of 30 and a maximum of 1000. For more details, see Table 3.

Table 3. Registered studies on diagnostics and measurement of disease activity.

\begin{tabular}{|c|c|c|c|c|c|c|}
\hline Objective & Methods Used & Study Type & $\begin{array}{l}\text { Registration } \\
\text { Number }\end{array}$ & Enroll. & $\begin{array}{l}\text { Age } \\
\text { (Years) }\end{array}$ & $\begin{array}{l}\text { Start } \\
\text { Year }\end{array}$ \\
\hline Physical activity & Indirect calorimetry & intervent. & NCT03913962 & 300 & $6-18$ & 2019 \\
\hline Physical activity & $\begin{array}{l}\text { Actigrafic } \\
\text { measurement }\end{array}$ & intervent. & NCT04167488 & 50 & $4-18$ & 2019 \\
\hline Physical activity & Exercise protocol & intervent. & NCT04671524 & 30 & $10-18$ & 2020 \\
\hline Spine deformities & $\begin{array}{l}\text { Trunk rotation } \\
\text { measurement and } \\
\text { radiological imaging }\end{array}$ & observ. & NCT04664231 & 200 & $4-16$ & 2021 \\
\hline $\begin{array}{l}\text { Differential diagnosis } \\
\text { of septic arthritis }\end{array}$ & $\begin{array}{l}\text { Proteic, cytokine and } \\
\text { cellular markers }\end{array}$ & observ. & NCT03827759 & 90 & $0-14$ & 2019 \\
\hline $\begin{array}{l}\text { Differential diagnosis } \\
\text { of septic arthritis }\end{array}$ & Mass spectrometry & observ. & NCT04460144 & 60 & $0-16$ & 2020 \\
\hline Imaging & MRI & observ. & NCT02684695 & 200 & $12-24$ & 2016 \\
\hline Imaging & Ultrasound & observ. & NCT04178837 & 33 & $1-17$ & 2020 \\
\hline $\begin{array}{l}\text { Temporomandibular } \\
\text { involvement }\end{array}$ & $\begin{array}{l}\text { Clinical examination, } \\
\text { CT }\end{array}$ & observ. & NCT02218892 & 62 & $7-14$ & 2011 \\
\hline $\begin{array}{l}\text { Temporomandibular } \\
\text { involvement, mouth } \\
\text { and teeth health }\end{array}$ & $\begin{array}{l}\text { DXA, MRI, caries } \\
\text { scores }\end{array}$ & observ. & NCT03904459 & 452 & $4-18$ & 2015 \\
\hline $\begin{array}{l}\text { sJIA and adult-onset } \\
\text { Stills disease }\end{array}$ & $\begin{array}{l}\text { History, physical } \\
\text { examination, } \\
\text { laboratory including } \\
\text { whole genome } \\
\text { sequencing }\end{array}$ & observ. & NCT03510442 & 1000 & 0-99 & 2018 \\
\hline $\begin{array}{l}\text { Disease activity } \\
\text { biomarker }\end{array}$ & $\begin{array}{l}\text { JADAS score } \\
\text { correlation with } \\
(1-3)-\beta-D-G l u c a n \\
\text { serum levels }\end{array}$ & intervent. & NCT03984669 & 40 & $0-18$ & 2019 \\
\hline $\begin{array}{l}\text { National disease } \\
\text { registry }\end{array}$ & $\begin{array}{l}\text { Longitudinal data } \\
\text { aquisition }\end{array}$ & observ. & NCT03245801 & 300 & $0-18$ & 2017 \\
\hline
\end{tabular}

\subsubsection{Medication Other Than DMARDs}

A total of $9 / 72$ studies $(12.5 \%)$ were related to medications other than DMARD and all of them of the interventional type, concerning the following medications: anti-IFNgamma in systemic JIA, genicular nerve block, high-dose nicotinamide, dexmedetomidine, mesenchymal stromal cells, ondansetron, probiotics, recombinant interleukine-2, and triamcinolone hexacetonide. Except for the study on anti-IFN-gamma, studies were academia-sponsored and located in a single country. Median enrollment was 104, with a minimum of 6 and a maximum of 202. See Table 4 for more details. 
Table 4. Registered studies on medication other than DMARDs.

\begin{tabular}{|c|c|c|c|c|c|c|}
\hline Objective & Comments & Phase & $\begin{array}{l}\text { Registration } \\
\text { Number }\end{array}$ & Enroll. & $\begin{array}{c}\text { Age } \\
\text { (Years) }\end{array}$ & $\begin{array}{l}\text { Start } \\
\text { Year }\end{array}$ \\
\hline Anti-IFN-gamma mAb & sJIA and MAS/sHLH & II & NCT03311854 & 10 & $0-18$ & 2018 \\
\hline Genicular nerve block & Against triamcinolone & IV & NCT04687930 & 104 & $0-16$ & 2020 \\
\hline $\begin{array}{l}\text { High dose } \\
\text { nicotinamide }\end{array}$ & $\begin{array}{l}\text { Penetration in synovial } \\
\text { fluid }\end{array}$ & $\mathrm{I} / \mathrm{II}$ & $\begin{array}{c}\text { EudraCT2018- } \\
\text { 002245-11 }\end{array}$ & 6 & $12-17$ & 2019 \\
\hline $\begin{array}{l}\text { Intranasal } \\
\text { dexmedetomidine }\end{array}$ & $\begin{array}{l}\text { Sedation for joint } \\
\text { injections }\end{array}$ & IV & NCT03069638 & 109 & $1-18$ & 2017 \\
\hline $\begin{array}{l}\text { Mesenchymal stromal } \\
\text { cells }\end{array}$ & In drug resistent JIA & $\mathrm{I} / \mathrm{II}$ & $\begin{array}{l}\text { EudraCT2012- } \\
\text { 002067-10 }\end{array}$ & 6 & $2-17$ & 2014 \\
\hline Ondansetron & Premedication for MTX & $\mathrm{n} / \mathrm{a}$ & NCT04169828 & 176 & $4-16$ & 2019 \\
\hline Probiotics & $\begin{array}{l}\text { Dietary } \\
\text { supplementation }\end{array}$ & $\mathrm{n} / \mathrm{a}$ & NCT03092427 & 120 & $1-7$ & 2017 \\
\hline Recombinant IL-2 & $\begin{array}{l}\text { JIA and other } \\
\text { rheumatic diseases }\end{array}$ & II & NCT04397107 & 46 & $0-17$ & 2020 \\
\hline $\begin{array}{l}\text { Triamcinolone } \\
\text { hexacetonide }\end{array}$ & Personalised treatment & IV & NCT04614311 & 202 & $1-18$ & 2020 \\
\hline
\end{tabular}

\subsubsection{Non-Medication Treatment}

A total of $6 / 72$ studies (8.3\%) were related to non-medication treatment of JIA and of the interventional type. Four behavioral intervention studies concerned sleep and/or pain self-management of JIA patients, and a dietary intervention with specific carbohydrates was performed in one other, as well as a peer mentoring program for adolescents with JIA for acquisition of transition preparedness. All of these studies were academia-sponsored and located in a single country. Median enrollment was 30, with a minimum of 18 and a maximum of 262. See Table 5 for more details.

Table 5. Registered studies on non-medication treatment.

\begin{tabular}{|c|c|c|c|c|c|}
\hline Objective & Comments & $\begin{array}{l}\text { Registration } \\
\text { Number }\end{array}$ & Enroll. & $\begin{array}{l}\text { Age } \\
\text { (Years) }\end{array}$ & $\begin{array}{l}\text { Start } \\
\text { Year }\end{array}$ \\
\hline Pain management & $\begin{array}{l}\text { Behavorial intervention } \\
\text { through yoga and aerobic } \\
\text { dance }\end{array}$ & NCT03833609 & 25 & $13-18$ & 2019 \\
\hline $\begin{array}{l}\text { Pain and sleep } \\
\text { management }\end{array}$ & Sleep manipulation protocol & NCT04133662 & 30 & $12-18$ & 2018 \\
\hline Sleep management & $\begin{array}{l}\text { Web-based program for } \\
\text { parents }\end{array}$ & NCT04066205 & 60 & $8-13$ & 2019 \\
\hline $\begin{array}{l}\text { Sleep management for } \\
\text { pre-schoolers }\end{array}$ & $\begin{array}{l}\text { Web-based program for } \\
\text { parents }\end{array}$ & NCT04354337 & 18 & $2-5$ & 2020 \\
\hline $\begin{array}{l}\text { Specific Carbohydrate } \\
\text { Diet }\end{array}$ & $\begin{array}{l}\text { As add-on therapy to } \\
\text { low-medium disease activity }\end{array}$ & NCT04205500 & 30 & $0-16$ & 2017 \\
\hline Transition preparedness & $\begin{array}{l}\text { Peer mentorship through } \\
\text { Skype calls }\end{array}$ & NCT03116763 & 262 & $12-18$ & 2017 \\
\hline
\end{tabular}

\subsubsection{Vaccination}

A total of $2 / 72$ studies $(2.8 \%)$ were related to JIA and vaccination: one observational study concerned the frequency of the human papilloma virus vaccination among JIA patients (NCT04180228), and one interventional study concerned the safety and efficacy of a live attenuated measles, mumps, and rubella vaccine in JIA patients (EudraCT2007-00186114). Both studies were academia-sponsored and located in France and the Netherlands, respectively. Enrollment was 150 and 280, respectively.

\subsubsection{COVID-19 Pandemic}

One observational, academia-sponsored study (1.4\%) was related to the COVID19 sanitary crisis and observed the impact on therapeutic management of JIA patients (NCT04407923). Localized in France, its enrollment was 150. 


\section{Discussion}

Current clinical research in JIA is mainly focused on drug therapy—which predominantly means DMARD agents and sponsoring by the pharmaceutical industry-followed by studies on diagnostics and measurement of disease activity. Non-medication therapy and other issues were clearly secondary. In general, the size of interventional clinical studies was relatively small, with a maximum enrollment of 340 participants. The two main study locations were Europe and North America, followed by South America and Asia.

\subsection{Role of Sponsor in Clinical Studies}

Ongoing registered clinical studies were sponsored by academia in about $60 \%$ of cases, and by (the pharma) industry in about $40 \%$. Industry-sponsored studies were doubleregistered in both registries in slightly more than half of the cases, which is not common for academia-sponsored studies.

Industry-sponsored studies almost exclusively studied DMARDs, except for one study that concerned treatment with anti-IFN gamma in systemic JIA. Only two of the industrysponsored studies were observational (pharmacovigilance on MTX, ABA, ADA) while most others were interventional and tested DMARDs, namely BAR, CER, IXE, SAR, SEC, TOF, and UPA, for introduction into treatment of JIA. Typically, drugs had recently been labeled for rheumatic or chronic inflammatory bowel diseases in adults first, and use was then explored for JIA patients [16]. Only a few interventional studies sponsored by the industry concerned longer established DMARDs in JIA, TOC above all. All industry-sponsored studies had localizations in more than one country; we assume that this might be due to greater access to potential participants, as well as to potential pharma markets.

Academia-sponsored studies did have much more varied objectives. Most of the observational studies (14/16) were conducted by academia, especially in the fields of diagnostics and disease activity measurement. In fact, at least one third of research in DMARDs is performed by academia, on longer established drugs including csDMARDs. Interestingly, withdrawal strategies in DMARD-treated patients play a significant role. Besides treatment with DMARDs, academia explores other possibilities of JIA treatment, including non-DMARD medications and non-medication (behavioral) treatment strategies. Multi-lateral localizations were an exceptional condition here; we assume that, in many cases, barriers between heterogenous legal areas increase the necessary effort for the realization of multilateral collaboration beyond feasibility for academia.

\subsection{Role of Novel DMARDs in Clinical Studies}

Unsurprisingly, in clinical studies in the field of JIA, DMARDs are the big player. The introduction of biological and targeted synthetic DMARDs have tremendously changed the game up to today $[2,4,7]$. The longer goal of treatment is not only to improve complaints and disabilities, but also to achieve complete disease control for the best long-term outcome. The most frequent targets in DMARD treatment (by the count of registered studies and enrollments) are TNF, JAK, IL-6, and T cell. Of these, JAK inhibitors are the most recently introduced class of pharmaceutics to JIA. Baricitinib and Tofacitinib have comparable study numbers and enrollments in current research, as earlier introduced TNF inhibitors. In contrast, similar novel IL-17 inhibitors (IXE, SEC) are tested in distinctly fewer studies with smaller enrollments, which might be due to a limited application field in ERA and JPsA concerning JIA. Few novel agents were tested for targets with longer available bDMARDs, namely TNF (Certolizumab, phase III) and IL-6 inhibitors (Sarilumab, phase II). Furthermore, studies on IL-1 antagonist agents had a smaller part in DMARD studies, which are relevant only for systemic JIA in the field of JIA, besides other indications such as hereditary fever syndromes.

Although the number of studies and total enrollments may be seen as markers for potential pharmacological candidates in general, clinical studies in children should limit the sample size needed and/or limit exposure to a placebo, such as through extrapolation of efficacy or early escape rules [22,23]. Rapidly expanding pharmacological options demand 
innovative clinical trial methods for accelerated authorization and introduction to JIA, and must not (only) rely on large sample sizes [24].

\subsection{Targeting Specific Issues in JIA}

The number of current studies on specific pediatric issues-besides more pharmacological options-is low. Musculoskeletal impairment is addressed in only a few studies, while growth disturbances and pubertal disorders are not. Frequent prevalent issues in JIA, such as temporomandibular involvement and JIA-associated uveitis, were found to be specific objectives in only a few clinical studies in this study. Restrictively, this study did not search for uveitis-specific studies, as only studies that refer to the term 'JIA' were evaluated. Family-centered care, social integration, and rehabilitation, as well as transition were not found to be explicit issues in ongoing studies. Potential answers to the question of why these issues were not, or were scarcely, found in current studies are: a negation of research need where an improved arsenal of therapeutics is available; challenges in designing appropriate studies; or that the choice of two clinical trial registries for evaluation might not capture the bulk of non-medication trials.

Most of the studies included several subgroups of JIA, mainly non-systemic forms or poly-/oligoarticular courses of JIA. Nevertheless, systemic JIA was specifically addressed in 12/72 studies (ten concerning DMARDs). Likewise, etiologically differing entities such as psoriatic and enthesitis-related arthritis were specifically addressed in 4/72 studies (all on DMARDs).

\subsection{Does Clinical Research Meet the Need for Research in JIA?}

It is not surprising that the majority (more than three quarters) of ongoing studies investigates particular treatment strategies on JIA. The value of scientific networking and collaboration, which brings research results into practice through guidelines and on-site rheumatologic care providers, can barely be shown by analyzing registered clinical studies. Specific issues of JIA, and pediatrics in general, were scarcely addressed. Transition into a vulnerable life stage is especially important for long-term outcomes, and of relevance to chronic diseases in pediatrics in general [25], particularly JIA, including somatic and mental health $[19,26,27]$. A direct reference to adolescents specifically, for instance, was only found in one of the studies, although in the context of peer mentoring.

\subsection{Limitations of This Analysis}

This study has several limitations. We used two registries (clinicaltrials.gov and clinicaltrialsregister.eu) whereby studies registered in smaller national registries, as well as from central registries outside Europe and North America, would be missing. Naturally, our study cannot determine studies and research that are not registered in any registry of clinical studies; this may be the case, especially for non-medication and/or observational studies. Our analyses rely on the accuracy of data input to these two registries. For the purpose of characterizing ongoing studies, we did not consider studies that were finally closed for further recruitment. Furthermore, we did not search for specific terms, i.e., uveitis, which may have revealed more research in these specific fields.

This study did not formally analyze design and methodology of the clinical studies presented here, such as inclusion/exclusion criteria, study duration, endpoints and efficacy assessment. The European Medicines Agency has issued a "guideline on clinical investigation of medicinal products for the treatment of juvenile idiopathic arthritis", which can be helpful for investigators [23]. In addition, innovative clinical trial methods and open dialogues between key stakeholders, such as investigators, regulators, and the pharmaceutical industry, may facilitate meaningful clinical research in the changing drug development landscape in JIA [24]. We consider this study a cross-sectional snapshot on the ongoing research in JIA in general, and not a specific in-depth exploration on research in predefined subsets. 


\section{Conclusions}

While clinical research is mainly focused on drug therapy and diagnostics, other issues in JIA management are marginal topics in registered studies. Drug development was transferred from adult rheumatology into the JIA population with little innovation for children. Future research should take specific pediatric needs better into account.

Supplementary Materials: The following are available online at https:/ / www.mdpi.com/article/ 10.3390/biomedicines9121860/s1. Supplementary File S1: Analyzed data (xlsx file).

Author Contributions: R.L. conducted the data analysis and interpretation, and wrote the draft of this manuscript. M.R. conceived of the study design, supervised data interpretation and revised the manuscript. All authors have read and agreed to the published version of the manuscript.

Funding: This research received no external funding.

Institutional Review Board Statement: Not applicable.

Data Availability Statement: The underlying data of this study are fully available as Supplementary Materials.

Conflicts of Interest: The authors declare no conflict of interest.

\section{Abbreviations}

\begin{tabular}{|c|c|}
\hline ABA & abatacept \\
\hline ADA & adalimumab \\
\hline ANA & anakinra \\
\hline BAR & baricitinib \\
\hline bDMARD & biological DMARD \\
\hline CAN & canakinumab \\
\hline CER & certolizumab \\
\hline csDMARD & conventional synthetic DMARD \\
\hline DMARD & disease modifying anti-rheumatic drug \\
\hline ERA & enthesitis related arthritis \\
\hline ETA & etanercept \\
\hline GOL & golimumab \\
\hline HCQ & hydroxychloroquin \\
\hline IFN & interferone \\
\hline IL & interleukine \\
\hline IXE & ixekizumab \\
\hline JAK & janus kinase \\
\hline JIA & juvenile idiopathic arthritis \\
\hline JPsA & juvenile psoriatic arthritis \\
\hline MAS/sHLH & $\begin{array}{l}\text { macrophage activation syndrome/secondary haemophagocytic } \\
\text { lymphohistiocytosis }\end{array}$ \\
\hline MTX & methotrexate \\
\hline $\mathrm{n} / \mathrm{a}$ & not available \\
\hline SAR & sarilumab \\
\hline SEC & secukinumab \\
\hline sJIA & systemic JIA \\
\hline SUL & sulfasalazine \\
\hline TNF & tumor necrosis factor \\
\hline TOC & tocilizumab \\
\hline TOF & tofacitinib \\
\hline tsDMARD & targeted synthetic DMARD \\
\hline UPA & upadacitinib \\
\hline
\end{tabular}




\section{References}

1. Ravelli, A.; Martini, A. Juvenile idiopathic arthritis. Lancet 2007, 369, 767-778. [CrossRef]

2. Bridges, J.M.; Mellins, E.D.; Cron, R.Q. Recent progress in the treatment of non-systemic juvenile idiopathic arthritis. Fac. Rev. 2021, 10, 23. [CrossRef] [PubMed]

3. Prakken, B.; Albani, S.; Martini, A. Juvenile idiopathic arthritis. Lancet 2011, 377, 2138-2149. [CrossRef]

4. Saougou, I.G.; Markatseli, T.E.; Voulgari, P.V.; Drosos, A.A. Current therapeutic options for the treatment of juvenile idiopathic arthritis. Curr. Rheumatol. Rev. 2020. [CrossRef]

5. D'Angelo, D.M.; Di Donato, G.; Breda, L.; Chiarelli, F. Growth and puberty in children with juvenile idiopathic arthritis. Pediatr. Rheumatol. Online J. 2021, 19, 28. [CrossRef] [PubMed]

6. Covert, L.; Mater, H.V.; Hechler, B.L. Comprehensive Management of Rheumatic Diseases Affecting the Temporomandibular Joint. Diagnostics 2021, 11, 409. [CrossRef]

7. Hashkes, P.J. 50 Years Ago in The Journal of Pediatrics: Revolutionary Changes in the Management of Juvenile Idiopathic Arthritis. J. Pediatr. 2020, 224, 65. [CrossRef]

8. Ruperto, N.; Martini, A. Current and future perspectives in the management of juvenile idiopathic arthritis. Lancet Child Adolesc. Health 2018, 2, 360-370. [CrossRef]

9. Klein, A.; Minden, K.; Hospach, A.; Foeldvari, I.; Weller-Heinemann, F.; Trauzeddel, R.; Huppertz, H.I.; Horneff, G. Treat-to-target study for improved outcome in polyarticular juvenile idiopathic arthritis. Ann. Rheum. Dis. 2020, 79, 969-974. [CrossRef]

10. Ringold, S.; Angeles-Han, S.T.; Beukelman, T.; Lovell, D.; Cuello, C.A.; Becker, M.L.; Colbert, R.A.; Feldman, B.M.; Ferguson, P.J.; Gewanter, H.; et al. 2019 American College of Rheumatology/Arthritis Foundation Guideline for the Treatment of Juvenile Idiopathic Arthritis: Therapeutic Approaches for Non-Systemic Polyarthritis, Sacroiliitis, and Enthesitis. Arthritis Rheumatol. 2019, 71, 846-863. [CrossRef]

11. Horneff, G.; Klein, A.; Ganser, G.; Sailer-Höck, M.; Günther, A.; Foeldvari, I.; Weller-Heinemann, F. Protocols on classification, monitoring and therapy in children's rheumatology (PRO-KIND): Results of the working group Polyarticular juvenile idiopathic arthritis. Pediatr. Rheumatol. Online J. 2017, 15, 78. [CrossRef] [PubMed]

12. Hinze, C.H.; Holzinger, D.; Lainka, E.; Haas, J.P.; Speth, F.; Kallinich, T.; Rieber, N.; Hufnagel, M.; Jansson, A.F.; Hedrich, C.; et al. Practice and consensus-based strategies in diagnosing and managing systemic juvenile idiopathic arthritis in Germany. Pediatr. Rheumatol. Online J. 2018, 16, 7. [CrossRef]

13. Choida, V.; Hall-Craggs, M.; Jebson, B.R.; Fisher, C.; Leandro, M.; Wedderburn, L.R.; Ciurtin, C. Biomarkers of Response to Biologic Therapy in Juvenile Idiopathic Arthritis. Front. Pharmacol. 2020, 11, 635823. [CrossRef] [PubMed]

14. Orczyk, K.; Smolewska, E. The Potential Importance of MicroRNAs as Novel Indicators How to Manage Patients with Juvenile Idiopathic Arthritis More Effectively. J. Immunol. Res. 2021, 2021, 9473508. [CrossRef]

15. Diener, C.; Horneff, G. Comparison of adverse events of biologicals for treatment of juvenile idiopathic arthritis: A systematic review. Expert Opin. Drug Saf. 2019, 18, 719-732. [CrossRef] [PubMed]

16. Singh, R.; Ivaturi, V.D.; Penzenstadler, J.; Liu, T.; Chen, J.; Marathe, A.; Ji, P.; Glaser, R.; Nikolov, N.; Sahajwalla, C. Response similarity assessment between polyarticular juvenile idiopathic arthritis and adult rheumatoid arthritis for biologics. Clin. Pharmacol. Ther. 2021. [CrossRef]

17. Chausset, A.; Pereira, B.; Echaubard, S.; Merlin, E.; Freychet, C. Access to paediatric rheumatology care in juvenile idiopathic arthritis: What do we know? A systematic review. Rheumatology 2020, 59, 3633-3644. [CrossRef]

18. Consolaro, A.; Giancane, G.; Alongi, A.; van Dijkhuizen, E.H.P.; Aggarwal, A.; Al-Mayouf, S.M.; Bovis, F.; De Inocencio, J.; Demirkaya, E.; Flato, B.; et al. Phenotypic variability and disparities in treatment and outcomes of childhood arthritis throughout the world: An observational cohort study. Lancet Child Adolesc. Health 2019, 3, 255-263. [CrossRef]

19. McColl, J.; Semalulu, T.; Beattie, K.A.; Alam, A.; Thomas, S.; Herrington, J.; Gorter, J.W.; Cellucci, T.; Garner, S.; Heale, L.; et al. Transition Readiness in Adolescents with Juvenile Idiopathic Arthritis and Childhood-Onset Systemic Lupus Erythematosus. ACR Open Rheumatol. 2021. [CrossRef]

20. Vandenbroucke, J.P.; von Elm, E.; Altman, D.G.; Gøtzsche, P.C.; Mulrow, C.D.; Pocock, S.J.; Poole, C.; Schlesselman, J.J.; Egger, M. Strengthening the Reporting of Observational Studies in Epidemiology (STROBE): Explanation and elaboration. Int. J. Surg. 2014, 12, 1500-1524. [CrossRef]

21. Krippendorff, K. Content Analysis: An Introduction to Its Methodology, 2nd ed.; Sage: Thousand Oaks, CA, USA, 2004.

22. European Medicines Agency. Concept Paper on Extrapolation of Efficacy and Safety in Medicine Development. Available online: https:/ / www.ema.europa.eu/en/documents/scientific-guideline/concept-paper-extrapolation-efficacy-safety-medicinedevelopment_en.pdf (accessed on 26 November 2021).

23. European Medicines Agency. Guideline on Clinical Investigation of Medicinal Products for the Treatment of Juvenile Idiopathic Arthritis (europa.eu). Available online: https://www.ema.europa.eu/en/documents/scientific-guideline/guideline-clinicalinvestigation-medicinal-products-treatment-juvenile-idiopathic-arthritis_en.pdf (accessed on 26 November 2021).

24. Schanberg, L.E.; Ramanan, A.V.; De Benedetti, F.; Beukelman, T.; Eakin, G.S.; Del Gaizo, V.; Ringold, S.; Vesely, R.; Schrandt, S.; Jaki, T.; et al. Toward Accelerated Authorization and Access to New Medicines for Juvenile Idiopathic Arthritis. Arthritis Rheumatol. 2019, 71, 1976-1984. [CrossRef] [PubMed]

25. Lestishock, L.; Nova, S.; Disabato, J. Improving Adolescent and Young Adult Engagement in the Process of Transitioning to Adult Care. J. Adolesc. Health 2021. [CrossRef] [PubMed] 
26. Palman, J.; McDonagh, J.E. Young Minds: Mental Health and Transitional Care in Adolescent and Young Adult Rheumatology. Open Access Rheumatol. 2020, 12, 309-321. [CrossRef]

27. McDonagh, J.E.; Farre, A. Transitional Care in Rheumatology: A Review of the Literature from the Past 5 Years. Curr. Rheumatol. Rep. 2019, 21, 57. [CrossRef] [PubMed] 